\title{
Direct and indirect searches for top-Higgs FCNC couplings
}

\author{
Hoda Hesari, ${ }^{1}$ Hamzeh Khanpour, ${ }^{2,1}$ and Mojtaba Mohammadi Najafabadi ${ }^{1}$ \\ ${ }^{1}$ School of Particles and Accelerators, Institute for Research in Fundamental Sciences, \\ P.O. Box 19395-5531, Tehran, Iran \\ ${ }^{2}$ Department of Physics, University of Science and Technology of Mazandaran, \\ P.O. Box 48518-78195, Behshahr, Iran \\ (Received 3 September 2015; published 29 December 2015)
}

\begin{abstract}
Large top-quark flavor changing through neutral currents is expected by many extensions of the standard model. Direct and indirect searches for flavor-changing neutral currents (FCNC) in the top-quark decays to an up-type quark (up, charm) and a Higgs boson are presented. We probe the observability of the top-Higgs FCNC couplings through the process $\mathrm{e}^{-} \mathrm{e}^{+} \rightarrow t\left(\rightarrow \ell \nu_{\ell} b\right) \bar{t}(\rightarrow q H)$, where $\ell=\mathrm{e}, \mu$ and $q$ reflects up and charm quarks. It is shown that the branching ratio $\operatorname{Br}(t \rightarrow q H)$ can be probed down to $1.12 \times 10^{-3}$ at $95 \%$ C.L. at the center-of-mass energy of $500 \mathrm{GeV}$ with an integrated luminosity of $3000 \mathrm{fb}^{-1}$. We also update the constraint on the top-Higgs FCNC coupling using the electroweak precision observables related to $Z \rightarrow c \bar{c}$ decay.
\end{abstract}

DOI: 10.1103/PhysRevD.92.113012

PACS numbers: $12.39 . \mathrm{Hg}, 13.85 . \mathrm{Rm}, 14.80 . \mathrm{Bn}, 14.65 . \mathrm{Ha}$

\section{INTRODUCTION}

The discovery of a Higgs boson with a mass of about $125 \mathrm{GeV}$ by the ATLAS and CMS experiments at the CERN-LHC $[1,2]$ has opened a window to search for new physics through precise measurements of the processes involving this particle. In particular, precise measurements of Higgs boson couplings to the standard model (SM) particles and its mass provide excellent opportunities for searches for the SM extensions. The Higgs boson mass and couplings to fermions and gauge bosons have been measured in various decay modes, and they are found to be in agreement with the predictions of the SM within uncertainties [3-7].

The top quark, the heaviest element of the SM, has the largest Yukawa coupling to the Higgs boson. With a mass of around $173.5 \mathrm{GeV}$, comparable to the electroweak symmetry-breaking scale, measurement of the top-quark properties would provide an appropriate probe for the electroweak symmetry-breaking mechanism. Within the $\mathrm{SM}$, the Higgs boson couples to fermions via Yukawa interactions, thereby producing the mass terms. There are no flavor-changing neutral current (FCNC) transitions mediated by the Higgs boson or by the $Z, \gamma, g$ gauge bosons at tree level. In other words, no leading-order transitions of $t \rightarrow q H$ or $t \rightarrow q V$, where $q$ reflects up or charm quarks and $V=\gamma, Z, g$, exist in the SM framework. The SM contributions to the top-quark FCNC occur at loop level, with the expected branching ratios around $10^{-15}-10^{-13}$ [8]. Such FCNC transitions are highly suppressed because of the Glashow-Iliopoulos-Maiani (GIM) mechanism [9], and top quarks almost exclusively decay to a bottom quark and a $W$ boson [10-12].

However, in some SM extensions, suppression due to the GIM mechanism can be relaxed because of the additional contributions of new particles in the loop diagrams, and consequently, larger branching ratios of $t \rightarrow q H$ or $t \rightarrow q V$ are expected. Quark singlet model [13,14], two-Higgs doublet models [15-20], the minimal supersymmetric standard model (MSSM) [21-26], extra dimensions [27], and natural composite Higgs models $[28,29]$ are examples of the SM extensions in which significant enhancements of top-quark FCNC appear. Even, in type III of the two-Higgs doublet model without flavor conservation, the $t \rightarrow q H$ transitions appear at tree level. These extensions of the SM can enhance the branching ratio of $t \rightarrow q H$ up to $10^{-5}$. Consequently, measuring any excess in the branching ratios for top-quark FCNC processes would be an indication of physics beyond the SM. There are already many studies on the probe of the FCNC processes and anomalous couplings in the top-quark sector in the literature [8,30-43].

Searches for the existence of physics beyond the SM can be performed either at high-energy colliders or using its indirect effects in higher-order processes. In this paper, we perform direct and indirect probes for the top-Higgs FCNC couplings. We redo the calculations which have been performed in Ref. [37] on the effects of top-Higgs FCNC couplings in the electroweak precision observables of the $Z$ boson and update the upper limit on $\operatorname{Br}(t \rightarrow c H)$ using the most recent measurements.

There are several proposals for a possible future $\mathrm{e}^{-} \mathrm{e}^{+}$ collider [44-50] which would provide precise measurements, in particular, in the top-quark sector and Higgs boson properties. As a direct way to search for the topHiggs FCNC interactions, we study the sensitivity of a future $\mathrm{e}^{-} \mathrm{e}^{+}$collider via $t \bar{t}$ events at center-of-mass energy of $500 \mathrm{GeV}$. We consider the case in which one of the top quarks decays to a $W$ boson and a bottom quark with leptonic decay of the $W$ boson $\left(t \rightarrow \ell \nu_{\ell} b\right)$ and the other top quark decays anomalously, $t \rightarrow q H(q=u$ and $c)$. We 
consider the $H \rightarrow b \bar{b}$ decay mode, as the Higgs boson decay into $b \bar{b}$ pairs has a maximum branching ratio [51], and high efficiency in tagging the jets originating from the hadronization of bottom quarks can be achieved $[46,52,53]$. We provide the $95 \%$ C.L. upper limit on the branching ratio of $t \rightarrow q H$ for various $b$-quark tagging efficiencies. There are several proposals for the center-of-mass energy and the integrated luminosity for a future electron-positron collider in the literature [54-57]. We give the results for the integrated luminosities of 300 and $3000 \mathrm{fb}^{-1}$ of data and the center-of-mass energy of $\sqrt{s}=500 \mathrm{GeV}$.

This paper is organized as follows. In Sec. II, we briefly describe the theoretical framework that we consider to study the top-Higgs FCNC interactions. In Sec. III, we review the current best limits on top-Higgs FCNC processes from direct and indirect searches. The update of the upper limit on $\operatorname{Br}(t \rightarrow c H)$ using electroweak precision observables of the $Z$ boson is also presented in Sec. III. In Sec. IV, we describe the Monte Carlo event generation, detector simulation for top-pair production in electronpositron collisions with FCNC decays of one of the top quarks $(t \rightarrow q H)$. The 95\% C.L. upper limits on the branching ratio of $t \rightarrow q H$ at different integrated luminosities and various $b$-tagging efficiencies are also presented in this section. Finally, our summary and conclusions are given in Sec. V.

\section{THEORETICAL FRAMEWORK}

The general effective Lagrangian describing the interaction of a light up-type quark $(q=u, c)$ with the top quark and a Higgs boson can be written as [58]

$$
\mathcal{L}=-\frac{g}{2 \sqrt{2}} \sum_{q=u, c} g_{t q H} \bar{q}\left(g_{t q H}^{v}+g_{t q H}^{a} \gamma_{5}\right) t H+\text { H.c. },
$$

where the dimensionless real coefficient $g_{t q H}$ (with $q=u$ and $c$ ) denotes the strength of the top-Higgs FCNC coupling, and $g$ is the weak coupling constant. The coefficients $g_{t q H}^{v}$ and $g_{t q H}^{a}$ are general complex numbers with the normalization $\left|g_{t q H}^{v}\right|^{2}+\left|g_{t q H}^{a}\right|^{2}=1$. Strong cancellations arising from the GIM mechanism cause a tiny value for $g_{t q H}$ in the SM. In the SM framework, $g_{t q H}$ amounts to $10^{-6}$, while in a big range of MSSM parameter space, a sizable value at the order of $10^{-2}$ is expected $[24,30]$.

After neglecting the up and charm quark masses, the $t \rightarrow q H$ and $t \rightarrow b W$ widths at leading order can be written as

$$
\begin{aligned}
& \Gamma(t \rightarrow q H)=\frac{\alpha}{32 s_{W}^{2}}\left|g_{t q H}\right|^{2} m_{t}\left[1-\frac{M_{H}^{2}}{m_{t}^{2}}\right]^{2}, \\
& \Gamma(t \rightarrow b W)=\frac{\alpha\left|V_{t b}\right|^{2}}{16 s_{W}^{2}} \frac{m_{t}^{3}}{m_{H}^{2}}\left(1-\frac{3 m_{W}^{4}}{m_{t}^{4}}+\frac{2 m_{W}^{6}}{m_{t}^{6}}\right),
\end{aligned}
$$

where $\alpha$ is the fine-structure constant, $V_{t b}$ is the CKM matrix element, $s_{W}$ is the sine of the Weinberg angle, and $m_{t}, m_{W}$, and $m_{H}$ are the top quark, $W$ boson, and Higgs boson masses, respectively. We estimate the branching ratio of $t \rightarrow q H$ as the ratio of $\Gamma(t \rightarrow q H)$ to the width of $t \rightarrow W b$. It has the following form:

$$
\begin{aligned}
\operatorname{Br}(t \rightarrow q H) & =\frac{g_{t q H}^{2}}{2} \frac{x^{2}}{1-3 x^{4}+2 x^{6}}\left(1-y^{2}\right)^{2} \\
& =0.0274 \times g_{t q H}^{2},
\end{aligned}
$$

where $x=m_{W} / m_{t}$ and $y=m_{H} / m_{t}$. For the calculations, we use $m_{H}=125.7 \mathrm{GeV}, m_{t}=173.21 \mathrm{GeV}, \alpha=1 / 128$, and $m_{W}=80.38 \mathrm{GeV}$ [59].

\section{CURRENT CONSTRAINTS ON $\mathrm{Br}(t \rightarrow q H)$}

In this section, we review the currently available limits on the branching ratio of $t \rightarrow q H$ from the collider experiments as well as the indirect limits. We also update the limits from observables related to $Z \rightarrow c \bar{c}$ decay.

Direct limits.-The ATLAS search for the $t q H \mathrm{FCNC}$ is based on the top-quark pair events with one-top-quark decays of $t \rightarrow q H(H \rightarrow \gamma \gamma)$ and the standard decays of the other top quark. The analysis uses $4.7 \mathrm{fb}^{-1}$ and $20.3 \mathrm{fb}^{-1}$ integrated luminosity of data collected at $\sqrt{s}=7$ and $8 \mathrm{TeV}$, respectively. Assuming $m_{H}=125.5 \mathrm{GeV}$, the observed limit on the branching ratio of $t \rightarrow q H$ at 95\% C.L. is $7.9 \times 10^{-3}$ [60]. This analysis has set an upper limit of $5.1 \times 10^{-3}$ at $95 \%$ C.L. on $\operatorname{Br}(t \rightarrow c H)$.

The limits from the CMS experiment are based on an inclusive search involving a lepton and a photon in the final state. The analysis uses $t \bar{t}$ events with one of the top quarks decaying to $c+H$ and standard model decays of the other top quark. The results correspond to $19.5 \mathrm{fb}^{-1}$ data at the center-of-mass energy of $8 \mathrm{TeV}$. The $95 \%$ C.L. upper limit on $\operatorname{Br}(t \rightarrow c H)$ was found to be $5.6 \times 10^{-3}$ for a Higgs boson mass of $126 \mathrm{GeV}$ [61]. Table I summarizes the current direct limits as well as the projected ones on the topHiggs FCNC branching ratios at the LHC and the High Luminosity LHC with a center-of-mass energy of $14 \mathrm{TeV}$ and with integrated luminosities of 300 and $3000 \mathrm{fb}^{-1}$. The LHC projections are taken from Ref. [62]. As can be seen from Table I, the best possible limit on $\operatorname{Br}(t \rightarrow q H)$ from the LHC would be at the order of $10^{-4}$ in the highluminosity regime.

Indirect limits. - Low-energy measurements in flavormixing processes can be used to constrain the top-quark flavor violation in the $t q H$ vertex. Considering higher order corrections, $D^{0}-\bar{D}^{0}$ mixing observable, the mass difference $\Delta M$, receives sizable contributions from both $t u H$ and $t c H$ couplings at the same time. Using the measured value of $\Delta M$, one can obtain a limit on the product of two couplings, i.e., $g_{t u H} g_{t c H}[63]$. With the Higgs boson mass in the range of 115$170 \mathrm{GeV}$, upper limits of $g_{t u H} g_{t c H} \leq(1.94-2.72) \times 10^{-2}$ 
TABLE I. Current direct limits as well as the projected ones on the $\operatorname{Br}(t \rightarrow q H)$ at the LHC and future HL-LHC.

\begin{tabular}{lccc}
\hline \hline Process & Br Limit & Search & Data set \\
\hline$t \rightarrow q H$ & $7.9 \times 10^{-3}$ & ATLAS $t \rightarrow t \rightarrow W b+q H \rightarrow \ell \nu b+\gamma \gamma q$ & $4.7,20 \mathrm{fb}^{-1} @ 7,8 \mathrm{TeV}$ \\
$t \rightarrow c H$ & $5.1 \times 10^{-3}$ & ATLAS $t \rightarrow t \rightarrow W b+q H \rightarrow \ell \nu b+\gamma \gamma q$ & $4.7,20 \mathrm{fb}^{-1} @ 7,8 \mathrm{TeV}$ \\
$t \rightarrow c H$ & $5.6 \times 10^{-3}$ & CMS $t \bar{t} \rightarrow W b+q H \rightarrow \ell \nu b+\ell \ell q X$ & $19.5 \mathrm{fb}^{-1} @ 8 \mathrm{TeV}$ \\
$t \rightarrow q H$ & $5 \times 10^{-4}$ & LHC $t \bar{t} \rightarrow W b+q H \rightarrow \ell \nu b+\gamma \gamma q$ & {$[60]$} \\
$t \rightarrow q H$ & $2 \times 10^{-4}$ & LHC $t \bar{t} \rightarrow W b+q H \rightarrow \ell \nu b+\gamma \gamma q$ & $300 \mathrm{fb}^{-1} @ 14 \mathrm{TeV}$ \\
$t \rightarrow q H$ & $2 \times 10^{-3}$ & LHC $t \bar{t} \rightarrow W b+q H \rightarrow \ell \nu b+\ell \ell q X$ & $3000 \mathrm{fb}^{-1} @ 14 \mathrm{TeV}$ \\
$t \rightarrow q H$ & $5 \times 10^{-4}$ & LHC $t \bar{t} \rightarrow W b+q H \rightarrow \ell \nu b+\ell \ell q X$ & $300 \mathrm{fb}^{-1} @ 14 \mathrm{TeV}$ \\
\hline \hline
\end{tabular}

are obtained. This corresponds to an upper limit of $\operatorname{Br}(t \rightarrow$ $q H)<(5.3-7.4) \times 10^{-4}$ if we assume $g_{t u H}=g_{t c H}$.

Another indirect way to constrain the top-Higgs FCNC couplings is to use the electroweak precision observables of $Z$ bosons [37]. The $t c H$ vertex contributes to the $Z \rightarrow c \bar{c}$ decay at loop level. It affects the electroweak precision observables in the $Z c \bar{c}$ vertex. The total width, partial width, and asymmetries are affected by the $t c H$ FCNC interaction. In [37], the $t c H$ vertex contribution has been calculated, and the upper limits of $\operatorname{Br}(t \rightarrow c H) \leq(0.09-$ $2.9) \times 10^{-3}$ for the Higgs mass in the range of $114 \leq m_{H} \leq$ $170 \mathrm{GeV}$ have been obtained. We update this limit with the Higgs boson mass of $125 \mathrm{GeV}$ using the current measurements of the $Z c \bar{c}$ vertex.

After taking into account the $t c H$ FCNC coupling contributions to the width of $Z \rightarrow c \bar{c}$, it can be written as

$$
\Gamma(Z \rightarrow c \bar{c})=\Gamma(Z \rightarrow c \bar{c})_{\mathrm{SM}}\left(1+\delta_{t c H}^{H}\right),
$$

where the $t c H$ one-loop corrections are given by $\delta_{t c H}^{H}$. The details of the calculations of $\delta_{t c H}^{H}$ are available in [37]. It can be expressed in terms of the Veltman-Passarino functions. Using the calculations and the related input from the Particle Data Group [64], an upper limit of $\operatorname{Br}(t \rightarrow c H) \leq$ $2.1 \times 10^{-3}$ is found at $95 \%$ C.L. As can be seen, the indirect limits are at the same order of the current direct limits, i.e., $10^{-3}$.

\section{STUDY OF $t q H$ IN TOP-PAIR EVENTS IN $\mathrm{e}^{-} \mathrm{e}^{+}$COLLISIONS}

In this section, we search for top-Higgs FCNC couplings in the $\mathrm{e}^{-} \mathrm{e}^{+} \rightarrow t\left(\rightarrow \ell \nu_{\ell} b\right) \bar{t}(\rightarrow q H)$ channel, where $\ell=\mathrm{e}$, $\mu$ and $q=u, c$, and we present the potential of a future electron-positron collider to probe tqH couplings. As mentioned before, we concentrate on the semileptonic decay of a top quark and the anomalous decay of another top quark with the Higgs boson decaying into a $b \bar{b}$ pair, as shown in Fig. 1. Therefore, the final state consists of an energetic lepton (muon or electron), a neutrino (appears as missing momentum), and four hadronic jets. Three of the jets are produced from the hadronization of bottom quarks.

In this study, we assume $g_{t q H}^{v}=1$ and no axial coupling, i.e., $g_{t q H}^{a}=0$. At the center-of-mass energy of $\sqrt{s}=500 \mathrm{GeV}$, the leading-order cross section including the branching ratios reads

$$
\sigma_{\sqrt{s}=500 \mathrm{GeV}}\left(g_{t q H}\right)=11.306 \times g_{t q H}^{2}(\mathrm{fb}) .
$$

At a higher center-of-mass energy, the cross section decreases as $1 / s$. Now, we turn to event generation and simulation. In order to simulate the signal events, the topHiggs FCNC effective Lagrangian [Eq. (1)] is implemented in the FeynRules package [65-67]; then, the model is imported to a Universal FeynRules Output (UFO) module [68]. After that, it is inserted into a MadGraph5aMC@NLO [69,70] event generator. PYTHIA [71,72] is utilized for parton showering and hadronization, and Delphes $3[73,74]$ is employed to account for detector effects.

The main background comes from top-pair events with semileptonic decay of one of the top quarks and hadronic decay of another top quark. Other backgrounds to our signal include $W^{ \pm} b \bar{b} j j, Z b \bar{b} j j$ (with leptonic decay of $Z$ ), and $Z \ell^{ \pm} \ell^{ \pm} j j$ (with hadronic decay of $Z$ ). The contribution of $W j j j j$, where $j$ denotes non-bottom-quark jets, is studied as well. All of these backgrounds are generated at leading order using MadGraph5-aMC@NLO.

To consider detector resolutions, the final-state particles, leptons and jets, are smeared according to Gaussian

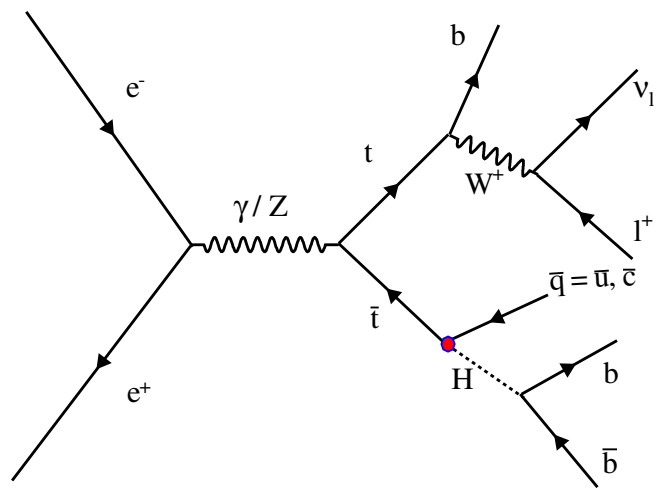

FIG. 1 (color online). The representative Feynman diagram for production of a $t \bar{t}$ event. It includes the decay chain with one leptonic top decay and the other top decay from anomalous FCNC coupling and Higgs decay into a $b \bar{b}$ pair. 
distributions using the following parametrizations, which are used in Delphes 3. Jet energies are smeared as $[47,75]$

$$
\frac{\Delta E_{j}}{E_{j}}=\frac{40.0 \%}{\sqrt{E_{j}}} \oplus 2.5 \% \text { (jets) }
$$

and for leptons (muons and electrons), we use a CMS-like detector resolution:

$$
\frac{\Delta E_{\ell}}{E_{\ell}}=\frac{7.0 \%}{\sqrt{E_{\ell}}} \oplus \frac{0.35}{E_{\ell}} \oplus 0.7 \% \text { (leptons), }
$$

where $E_{j}$ and $E_{\ell}$ represent the energies of the jets and leptons, respectively. The energies are in $\mathrm{GeV}$, and the symbol $\oplus$ represents a quadrature sum. It should be mentioned that the electron and muon energy resolutions are different; however, for simplicity, we smear the energies of muons similarly to the electrons.

The anti- $k_{t}$ algorithm [76] with a jet radius of 0.4 is used to reconstruct jets. We present the results for three $b$-jet identification efficiencies of $\epsilon_{b}=60 \%, 70 \%, 80 \%$. A mistag rate of $10 \%$ for charm-quark jets and $1 \%$ for other light-flavor jets are considered. It is notable that $b$-tagging efficiency and mistag rates play important roles in this analysis, as we have bjets in the final state as well as in light jets.

The events are selected according to the following strategy. For each event, to reconstruct the semileptonic decaying top quark, we require exactly one charged lepton with $p_{T}^{\ell}>10 \mathrm{GeV}$ within the pseudorapidity range of $\left|\eta^{\ell}\right|<2.5$. The events with more than one charged lepton are discarded. The $W$-boson momentum in the top-quark decay is obtained by summing the momenta of the charged lepton and neutrino. Each event is required to have exactly four jets, $n_{j}=4$, with $p_{T}^{\text {jets }}>20$ and $\left|\eta^{\text {jets }}\right|<2.5$. Among the jets, at least three jets must be $b$-tagged jets. The $b$-jet multiplicity is presented in Fig. 2 for signal and different

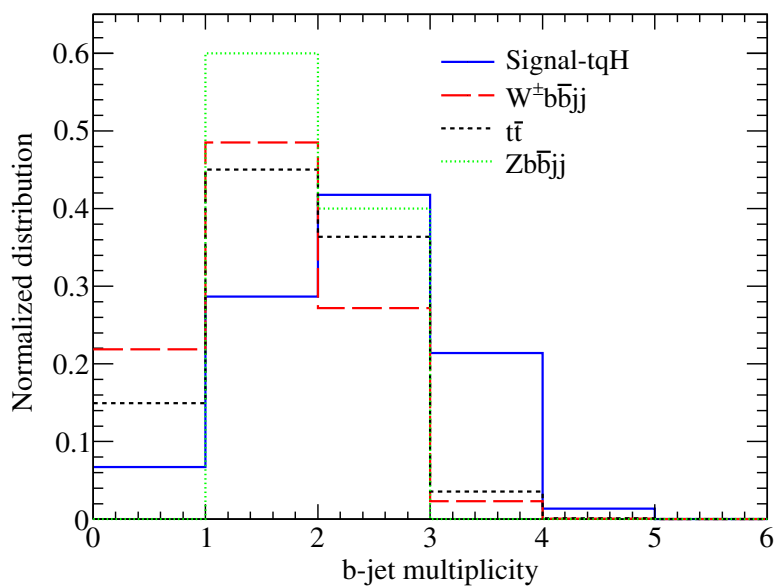

FIG. 2 (color online). Distribution of $b$-jet multiplicity for signal and SM backgrounds.
SM backgrounds. As can be seen from the distributions, the three $b$-tag jets requirement is considerably useful to reduce contributions of different backgrounds.

To have well-isolated objects, for any pair of objects in the final state, we require $\Delta R_{i j}=$ $\sqrt{\left(\eta_{i}-\eta_{j}\right)^{2}+\left(\phi_{i}-\phi_{j}\right)^{2}}>0.4$, with $i$ and $j$ running over all particles in the final state.

To reconstruct the Higgs boson and then both top quarks, there are ambiguities to choose the correct combinations of the $b$ jets. To solve the ambiguities and reconstruct the Higgs boson and $t \bar{t}$ system, we define $\chi^{2}$ as

$$
\chi_{b_{m} b_{n} b_{k}}^{2}=\left(m_{b_{m} W}-m_{\mathrm{top}}\right)^{2}+\left(m_{b_{n} b_{k}}-m_{\mathrm{Higgs}}\right)^{2} .
$$

Various combinations of $\chi_{b_{m} b_{n} b_{k}}^{2}$, where $m, n$, and $k$ run over the $b$ jets, are made, and the one with minimum $\chi^{2}$ is chosen. The mass distribution of the reconstructed Higgs boson is illustrated in Fig. 3. As can be seen, the invariant mass distribution peaks at the Higgs boson mass for signal events, while backgrounds have wide distributions. As a result, applying a mass window cut can reduce the background contributions. We require the reconstructed invariant mass of the Higgs boson to satisfy $90 \mathrm{GeV}<m_{\text {Higgs }}^{\text {reco }}<140 \mathrm{GeV}$.

Table II summarizes the cross sections (in fb) after applying the cuts for the signal and backgrounds. The $b$ tagging efficiency is assumed to be $60 \%$ in this table. The contribution of $Z b \bar{b} j j$ (with $Z \rightarrow \ell^{ \pm} \ell^{ \pm}$) and $Z \ell^{ \pm} \ell^{ \pm} j j$ (with $Z \rightarrow j j$ ) backgrounds is negligible after all cuts. After the jet requirements (set II of the cuts in Table II), the cross section is at the order of $10^{-5}$ and goes to zero after the three $b$-jet requirement. None of the $W^{ \pm} j j j j$ events, where $j$ denotes light flavor jets, survives after the three $b$-jet requirement. Therefore, these sources of backgrounds are not mentioned in Table II. Considering different sources of

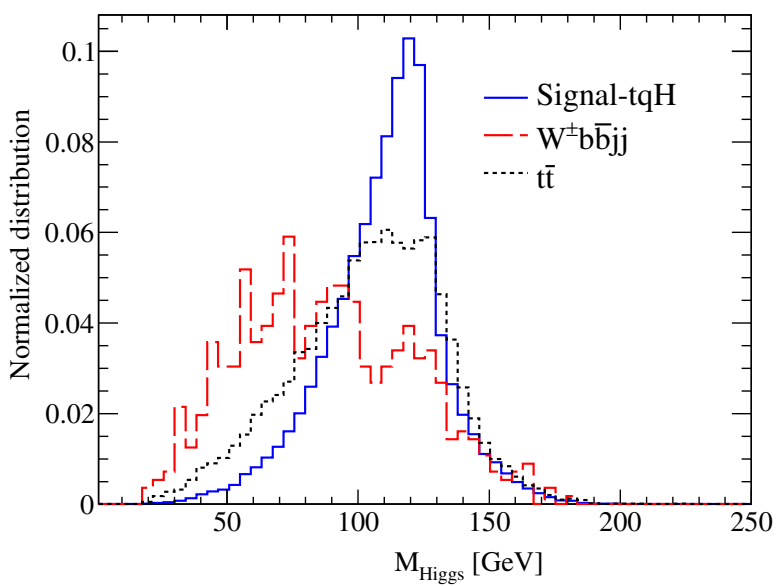

FIG. 3 (color online). The reconstructed Higgs boson mass distribution from the $\chi^{2}$ analysis for signal and backgrounds. The signal sample is generated with $g_{t u H}=0.5$. 
TABLE II. Cross sections (in fb) after applying different sets of cuts for signal and background. The $b$-tagging efficiency is assumed to be $60 \%$ in this table. The details of the basic cuts applied are presented in the text.

\begin{tabular}{lccc}
\hline \hline$\sqrt{s}=500 \mathrm{GeV}$ & Signal & \multicolumn{2}{c}{ Backgrounds } \\
\hline Cuts & $\sigma_{t q H}(\mathrm{fb})$ & $\sigma_{W^{ \pm} b \bar{b} j j}(\mathrm{fb})$ & $\sigma_{t \bar{t}}(\mathrm{fb})$ \\
No cut & $11.306\left(g_{t q H}\right)^{2}$ & 1.72 & 148.70 \\
$(\mathrm{I}): 1 \ell+\left|\eta^{\ell}\right|<2.5+P_{T}^{\ell}>10+E_{T}^{\text {miss }}>10$ & $7.972\left(g_{t q H}\right)^{2}$ & 1.623 & 106.065 \\
(II): 4 jets $+\left|\eta^{\text {jets }}\right|<2.5+P_{T}^{\text {jets }}>20+\Delta R_{\ell \text {,jets }} \geq 0.4$ & $3.399\left(g_{t q H}\right)^{2}$ & 0.0071 & 47.824 \\
(III): $n_{b-\text { jet }} \geq 3+\Delta R_{\ell, b-\text { jets }} \geq 0.4$ & $0.709\left(g_{t q H}\right)^{2}$ & 0.00015 & 1.417 \\
(IV): $90<m_{\text {Higgs }}^{\text {reco }}<140$ & $0.570\left(g_{t q H}\right)^{2}$ & 0.00005 & 0.961 \\
\hline \hline
\end{tabular}

TABLE III. The $95 \%$ C.L. limits on $\operatorname{Br}(t \rightarrow q H)$ for $b$-tagging efficiencies of $60 \%, 70 \%$, $80 \%$ with 300 and $3000 \mathrm{fb}^{-1}$ of integrated luminosity of data.

\begin{tabular}{lccc}
\hline \hline$b$-tagging efficiency & $\mathrm{IL}$ & Upper limit on $g_{t q H}$ & Upper limit on $\operatorname{Br}(t \rightarrow q H)$ \\
\hline$\epsilon_{b}=60 \%$ & $300 \mathrm{fb}^{-1}$ & 0.463 & $5.894 \times 10^{-3}$ \\
$\epsilon_{b}=60 \%$ & $3000 \mathrm{fb}^{-1}$ & 0.256 & $1.798 \times 10^{-3}$ \\
$\epsilon_{b}=70 \%$ & $300 \mathrm{fb}^{-1}$ & 0.373 & $3.821 \times 10^{-3}$ \\
$\epsilon_{b}=70 \%$ & $3000 \mathrm{fb}^{-1}$ & 0.202 & $1.126 \times 10^{-3}$ \\
$\epsilon_{b}=80 \%$ & $300 \mathrm{fb}^{-1}$ & 0.301 & $2.476 \times 10^{-3}$ \\
$\epsilon_{b}=80 \%$ & $3000 \mathrm{fb}^{-1}$ & 0.166 & $7.546 \times 10^{-4}$ \\
\hline \hline
\end{tabular}

systematic uncertainties in detail is beyond the scope of this work so an overall uncertainty of $30 \%$ is conservatively assigned to the number of background events for the limitsetting procedure.

Now, we proceed to set the $95 \%$ C.L. upper limit on the signal cross section. Then, the limits are translated into the upper limits on $\operatorname{Br}(t \rightarrow q H)$. Upper limits on the signal cross section are calculated with a $\mathrm{CL}_{s}$ approach [77]. The RooStats [78] program is utilized for the numerical evaluations of the $\mathrm{CL}_{s}$ limits.

We summarize the $95 \%$ C.L. limits on $\operatorname{Br}(t \rightarrow q H)$ in Table III for three $b$-tagging efficiencies of $60 \%, 70 \%, 80 \%$ with $300 \mathrm{fb}^{-1}$ and $3000 \mathrm{fb}^{-1}$ of integrated luminosity of data. With a $b$-tagging efficiency of $70 \%$ and $300 \mathrm{fb}^{-1}$ of data, an upper limit of $5.894 \times 10^{-3}$ could be achieved. As can be seen from Table III, higher $b$-tagging efficiency leads improved limits at the level of $30 \%-40 \%$. More data makes the upper limits better; however, the gain is less than 1 order of magnitude.

In comparison with the LHC direct limits presented in Table I, a future electron-positron collider would be able to reach similar sensitivity to the LHC experiments. The limits of the electron-positron collider could be significantly improved by including other decay modes of the Higgs boson such as $H \rightarrow \gamma \gamma, W^{+} W^{-}$, and ZZ. In addition, utilizing a more powerful tool, such as a multivariate technique, to separate signal from background could provide better sensitivity.

\section{SUMMARY AND CONCLUSIONS}

In this paper, we have presented direct and indirect searches for top-Higgs FCNC couplings. The radiative corrections due to $t c H$ coupling on the electroweak precision observables of $Z \rightarrow c \bar{c}$ decay are used to constrain $\operatorname{Br}(t \rightarrow c H)$ using the most recent measurements. This leads to the upper limit of $2.1 \times 10^{-3}$ on $\operatorname{Br}(t \rightarrow c H)$.

As a direct search, we study a future electron-positron collider potential at the center-of-mass energy of $500 \mathrm{GeV}$ to search for the $t q H$ FCNC couplings via top-quark pair production. The search is based on the process in which one of the top quarks decays leptonically $\left(t \rightarrow b \ell \nu_{\ell}\right)$ and the other top quark decays anomalously to $t \rightarrow q H$ with Higgs boson decays into $b \bar{b}$ pairs. The $95 \%$ C.L. upper limits on the branching ratio of $\operatorname{Br}(t \rightarrow q H)$ with $q=u$ and $c$ quarks are found to be $5.894 \times 10^{-3}$ for $300 \mathrm{fb}^{-1}$ of integrated luminosity of data. This upper limit decreases down to $1.798 \times 10^{-3}$ for the $3000 \mathrm{fb}^{-1}$ data. We find that $b$-tagging efficiency plays an essential role in this analysis and can improve the results at the level of 30\%-40\%, moving from an efficiency of $60 \%$ to $70 \%$. These limits could be considerably improved by including the other decay modes of the Higgs boson such as $\gamma \gamma, W^{+} W^{-}$, and $Z Z$.

\section{ACKNOWLEDGMENTS}

The authors thank R. Martinez for help in updating the limits from the $Z$-boson electroweak precision observables. Special thanks to $\mathrm{S}$. Khatibi for providing the FCNC model for simulating the events in MadGraph. The authors are thankful to the School of Particles and Accelerators, Institute for Research in Fundamental Sciences (IPM) for financial support of this project. H. K. also thanks the University of Science and Technology of Mazandaran for financial support provided for this research. 
[1] G. Aad et al. (ATLAS Collaboration), Phys. Lett. B 716, 1 (2012).

[2] S. Chatrchyan et al. (CMS Collaboration), Phys. Lett. B 716, 30 (2012).

[3] V. Khachatryan et al. (CMS Collaboration), Eur. Phys. J. C 75, 212 (2015).

[4] V. Khachatryan et al. (CMS Collaboration), Eur. Phys. J. C 75, 251 (2015).

[5] CMS Collaboration, Report No. CMS-PAS-HIG-13-001.

[6] CMS Collaboration, Report No. CMS-PAS-HIG-13-005.

[7] CMS Collaboration, Report No. CMS-PAS-HIG-14-007.

[8] J. A. Aguilar-Saavedra, Acta Phys. Pol. B 35, 2695 (2004).

[9] S. L. Glashow, J. Iliopoulos, and L. Maiani, Phys. Rev. D 2 , 1285 (1970).

[10] V. Khachatryan et al. (CMS Collaboration), Phys. Lett. B 736, 33 (2014).

[11] G. Eilam, J. L. Hewett, and A. Soni, Phys. Rev. D 44, 1473 (1991); 59, 039901(E) (1998).

[12] B. Mele, S. Petrarca, and A. Soddu, Phys. Lett. B 435, 401 (1998).

[13] F. del Aguila, J. A. Aguilar-Saavedra, and R. Miquel, Phys. Rev. Lett. 82, 1628 (1999).

[14] J. A. Aguilar-Saavedra and B. M. Nobre, Phys. Lett. B 553, 251 (2003).

[15] D. Atwood, L. Reina, and A. Soni, Phys. Rev. D 55, 3156 (1997).

[16] S. Bejar, arXiv:hep-ph/0606138.

[17] R. Gaitan, R. Martinez, and J. H. M. de Oca, arXiv: 1503.04391.

[18] G. Abbas, A. Celis, X. Q. Li, J. Lu, and A. Pich, arXiv:1503.06423.

[19] B. Altunkaynak, W. S. Hou, C. Kao, M. Kohda, and B. McCoy, Phys. Lett. B 751, 135 (2015).

[20] G. Abbas, A. Celis, X. Q. Li, J. Lu, and A. Pich, J. High Energy Phys. 06 (2015) 005.

[21] J. M. Yang, B. L. Young, and X. Zhang, Phys. Rev. D 58, 055001 (1998).

[22] G. M. de Divitiis, R. Petronzio, and L. Silvestrini, Nucl. Phys. B504, 45 (1997).

[23] J. L. Lopez, D. V. Nanopoulos, and R. Rangarajan, Phys. Rev. D 56, 3100 (1997).

[24] J. Guasch and J. Sola, Nucl. Phys. B562, 3 (1999).

[25] J. J. Liu, C. S. Li, L. L. Yang, and L. G. Jin, Phys. Lett. B 599, 92 (2004).

[26] J. J. Cao, G. Eilam, M. Frank, K. Hikasa, G. L. Liu, I. Turan, and J. M. Yang, Phys. Rev. D 75, 075021 (2007).

[27] K. Agashe, G. Perez, and A. Soni, Phys. Rev. D 75, 015002 (2007).

[28] K. Agashe and R. Contino, Phys. Rev. D 80, 075016 (2009).

[29] A. Azatov, G. Panico, G. Perez, and Y. Soreq, J. High Energy Phys. 12 (2014) 082.

[30] J. A. Aguilar-Saavedra and G. C. Branco, Phys. Lett. B 495, 347 (2000).

[31] G. Durieux, F. Maltoni, and C. Zhang, Phys. Rev. D 91, 074017 (2015).

[32] R. Goldouzian, Phys. Rev. D 91, 014022 (2015).

[33] Y. Wang, F. P. Huang, C. S. Li, B. H. Li, D. Y. Shao, and J. Wang, Phys. Rev. D 86, 094014 (2012).

[34] S. Khatibi and M. M. Najafabadi, Phys. Rev. D 89, 054011 (2014).
[35] J. L. Agram, J. Andrea, E. Conte, B. Fuks, D. Gel, and P. Lansonneur, Phys. Lett. B 725, 123 (2013).

[36] J. A. Aguilar-Saavedra, Nucl. Phys. B837, 122 (2010).

[37] F. Larios, R. Martinez, and M. A. Perez, Phys. Rev. D 72, 057504 (2005).

[38] H. Hesari, H. Khanpour, M. K. Yanehsari, and M. M. Najafabadi, Adv. High Energy Phys. 2014, 476490 (2014).

[39] H. Khanpour, S. Khatibi, M. K. Yanehsari, and M. M. Najafabadi, arXiv:1408.2090.

[40] N. Craig, J. A. Evans, R. Gray, M. Park, S. Somalwar, S. Thomas, and M. Walker, Phys. Rev. D 86, 075002 (2012).

[41] S. M. Etesami and M. Mohammadi Najafabadi, Phys. Rev. D 81, 117502 (2010).

[42] W. Bernreuther, D. Heisler, and Z. G. Si, arXiv:1508.05271.

[43] L. Wu, J. High Energy Phys. 02 (2015) 061.

[44] J. E. Brau, R. M. Godbole, F. R. L. Diberder, M. A. Thomson, H. Weerts, G. Weiglein, J. D. Wells, and H. Yamamoto, arXiv:1210.0202.

[45] T. Barklow, J. Brau, K. Fujii, J. Gao, J. List, N. Walker, and K. Yokoya, arXiv:1506.07830.

[46] H. Baer, T. Barklow, K. Fujii, Y. Gao, A. Hoang, S. Kanemura, J. List, H. E. Logan et al., arXiv:1306.6352.

[47] L. Linssen, A. Miyamoto, M. Stanitzki, and H. Weerts, arXiv:1202.5940.

[48] M. Aicheler, M. Aicheler, P. Burrows, M. Draper, T. Garvey, P. Lebrun, K. Peach, N. Phinney et al., Reports No. CERN2012-007, No. SLAC-R-985, No. KEK-Report-2012-1, No. PSI-12-01, and No. JAI-2012-001.

[49] M. Bicer et al. (TLEP Design Study Working Group Collaboration), J. High Energy Phys. 01 (2014) 164.

[50] M. Ahmad et al. (CEPC-SPPC Study Group), CEPC-SPPC Preliminary Conceptual Design Reports No. IHEP-CEPCDR-2015-01, No. IHEP-EP-2015-01, and No. IHEP-TH2015-01 (2015).

[51] A. Denner, S. Heinemeyer, I. Puljak, D. Rebuzzi, and M. Spira, Eur. Phys. J. C 71, 1753 (2011).

[52] H. Abramowicz et al. (CLIC Detector and Physics Study Collaboration), arXiv:1307.5288.

[53] T. Suehara and T. Tanabe, Nucl. Instrum. Methods Phys. Res., Sect. A 808, 109 (2016).

[54] D. M. Asner, T. Barklow, C. Calancha, K. Fujii, N. Graf, H. E. Haber, A. Ishikawa, S. Kanemura et al., arXiv: 1310.0763.

[55] S. Dawson, A. Gritsan, H. Logan, J. Qian, C. Tully, R. Van Kooten, A. Ajaib, A. Anastassov et al., arXiv:1310.8361.

[56] M. E. Peskin, arXiv:1312.4974.

[57] M. A. Fedderke, T. Lin, and L. T. Wang, arXiv:1506.05465.

[58] J. A. Aguilar-Saavedra, Nucl. Phys. B821, 215 (2009).

[59] K. A. Olive et al. (Particle Data Group Collaboration), Chin. Phys. C 38, 090001 (2014).

[60] G. Aad et al. (ATLAS Collaboration), J. High Energy Phys. 06 (2014) 008.

[61] CMS Collaboration, Report No. CMS-PAS-HIG-13-034.

[62] K. Agashe et al. (Top Quark Working Group Collaboration), arXiv:1311.2028.

[63] J. I. Aranda, A. Cordero-Cid, F. Ramirez-Zavaleta, J. J. Toscano, and E. S. Tututi, Phys. Rev. D 81, 077701 (2010).

[64] K. A. Olive et al. (Particle Data Group Collaboration), Chin. Phys. C 38, 090001 (2014). 
[65] A. Alloul, N. D. Christensen, C. Degrande, C. Duhr, and B. Fuks, Comput. Phys. Commun. 185, 2250 (2014).

[66] N. D. Christensen and C. Duhr, Comput. Phys. Commun. 180, 1614 (2009).

[67] C. Duhr and B. Fuks, Comput. Phys. Commun. 182, 2404 (2011).

[68] C. Degrande, C. Duhr, B. Fuks, D. Grellscheid, O. Mattelaer, and T. Reiter, Comput. Phys. Commun. 183, 1201 (2012).

[69] J. Alwall, R. Frederix, S. Frixione, V. Hirschi, F. Maltoni, O. Mattelaer, H.-S. Shao, T. Stelzer, P. Torrielli, and M. Zaro, J. High Energy Phys. 07 (2014) 079.

[70] J. Alwall, M. Herquet, F. Maltoni, O. Mattelaer, and T. Stelzer, J. High Energy Phys. 06 (2011) 128.
[71] T. Sjostrand, S. Mrenna, and P. Z. Skands, Comput. Phys. Commun. 178, 852 (2008).

[72] T. Sjostrand, L. Lonnblad, S. Mrenna, and P. Z. Skands, arXiv:hep-ph/0308153.

[73] J. de Favereau, C. Delaere, P. Demin, A. Giammanco, V. Lemaître, A. Mertens, and M. Selvaggi (DELPHES 3 Collaboration), J. High Energy Phys. 02 (2014) 057.

[74] A. Mertens, J. Phys. Conf. Ser. 608, 012045 (2015).

[75] J. Brau et al. (ILC Collaboration), arXiv:0712.1950.

[76] M. Cacciari, G. P. Salam, and G. Soyez, J. High Energy Phys. 04 (2008) 063.

[77] B. Mistlberger and F. Dulat, arXiv:1204.3851.

[78] L. Moneta et al., The RooStats Project, Proc. Sci., ACAT2010 (2010) 057 [arXiv:1009.1003]. 